\title{
An Improved Two-Vector Model Predictive Torque Control based on RMS Duty Ratio Optimization for PMSM
}

\author{
Chenwei Ma \\ Department of Electrical Energy, \\ Metals, Mechanical \\ Constructions \& Systems \\ Ghent University \\ EEDT-Decision and Control \\ Flanders Make \\ Ghent, Belgium \\ Institute of Electrical Engineering \\ Harbin Engineering University \\ Harbin, China \\ Chenwei.Ma@UGent.be
}

\author{
Xuliang Yao \\ Institute of Electrical Engineering \\ Harbin Engineering University \\ Harbin, China \\ yaoxuliang@hrbeu.edu.cn
}

\author{
Huayu Li \\ Department of Electrical Energy, \\ Metals, Mechanical \\ Constructions \& Systems \\ Ghent University \\ EEDT-Decision and Control \\ Flanders Make \\ Ghent, Belgium \\ Huayu.Li@UGent.be
}

\author{
Frederik De Belie \\ Department of Electrical Energy, \\ Metals, Mechanical \\ Constructions \& Systems \\ Ghent University \\ EEDT-Decision and Control \\ Flanders Make \\ Ghent, Belgium \\ Frederik.DeBelie@UGent.be
}

\begin{abstract}
This paper proposes an improved two-vector modelpredictive torque control (MPTC) strategy to reduce the average torque ripple and improve the flux tracking performance. When determining the duty ratio of vector combination, this method aims at restricting the root mean square (RMS) error of both torque and flux during the whole control period. Every vector combination and corresponding time duration are evaluated in the cost function, which leads to global restriction of torque ripple and flux ripple. In order to avoid increasing switching frequency and computational burden, a restriction is added on the second vector. The three candidates of the second vector are the two adjacent vectors of the first one and zero vector. Simulation results are provided to show the effectiveness of the proposed strategy.
\end{abstract}

Keywords-Permanent magnet synchronous motor, predictive control, root mean square, torque and flux ripple

\section{INTRODUCTION}

Permanent magnet synchronous machines (PMSMs) with advantages of high efficiency, high torque density and good dynamic performance, have been employed in many industrial areas [1-3]. Direct torque control (DTC) is one of the most popular control strategies for PMSM due to its fast dynamic response and simple structure [4]. However, the main drawbacks of DTC are high torque ripple and high flux ripple [5-6]. In recent years, finite-control-set model predictive torque control (FCS-MPTC), which is considered as an alternative control strategy to DTC has been widely researched. It takes the advantage of the inherent discrete nature of power converters, predicts the future state of the system and select the optimal voltage vector which can minimize a predefined cost function [7-9]. In spite of advantages above, torque ripple and flux ripple are still inevitable for MPTC, since only one vector is selected during a fixed full control period.

Recently, several researches aiming at reducing torque ripple of MPTC have been conducted. In [10], a tolerance band is set to restrict the torque. In one control period, an active vector is first selected based on the cost function. When the torque reaches the boundary of the tolerance band under the active vector, zero vector is applied for the rest of the control period. However, zero vector may not be the best choice for the second vector. In [11] and [12], the switching instant is determined based on solving optimization problem, instead of the torque boundary. Although the torque ripple is reduced, this method failed to take the duration of the active vector into consideration when determining the optimal vector, since the duration of the vector is modified when hitting the boundary. Authors of [13] propose a generalized two-vector-based MPTC method. In this method, the restriction of the second vector is released from zero vector to arbitrary vector. It means that $7 \times 7=49$ voltage vector combinations can be selected, which provides more possibilities to reduce torque ripple. However, the computational burden is remarkably increased. In addition, when determining the duration of the first vector, only the instantaneous minimum torque error at the end of the control period is considered, namely deadbeat (DB) control. It cannot guarantee that the mean torque ripple and the flux ripple are all reduced during the whole control period.

In this paper, an improved two-vector MPTC with root mean square (RMS) error duty ratio optimization for PMSM is proposed. This strategy utilizes two vectors in one control period to improve the steady state performance and eliminate the current harmonic. Meanwhile, a principle of RMS error is utilized when determining the duty ratio. It can restrict the average torque and flux error over the whole control period. Besides, better flux tracking performance can be reached. In this strategy, duty ratio is calculated for every vector combination before selection. It guarantees the optimal vector combination with corresponding duration can be selected when evaluating the cost function. Furthermore, to reach a balance among switching frequency, computational burden and steady state performance, a restriction is set on the second vector. Two adjacent vectors of 


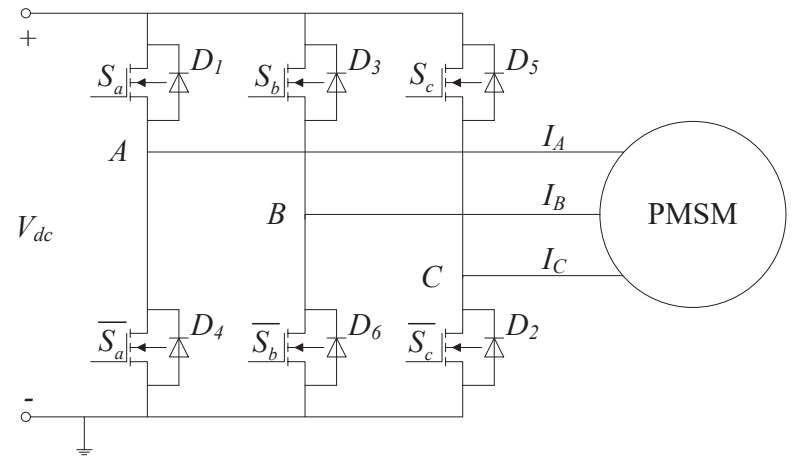

Fig. 1. Two level VSI.

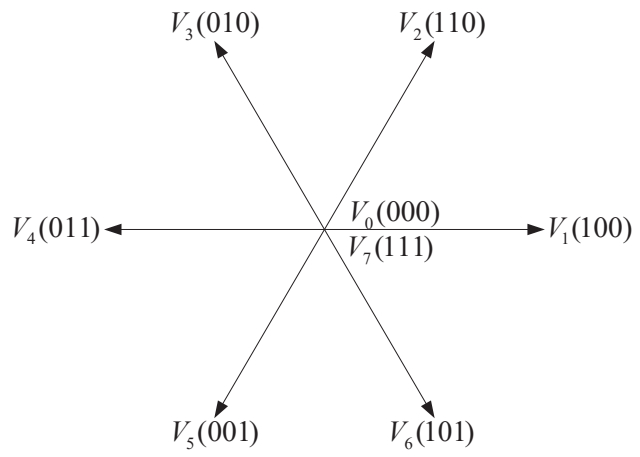

Fig. 2. Possible voltage vectors.

the first vector and zero vector are regarded as the candidates for the second vector. The simulation results show that better steady state performance are achieved in the proposed strategy compared with the conventional FCS-MPTC and the conventional two-vector MPTC method.

\section{MODEL OF PMSM AND INVERTER}

To analyze the MPTC strategy, this paper uses the rotary reference frame. Hence, the equations of surface mounted $\operatorname{PMSM}\left(L_{d}=L_{q}=L_{s}\right)$ in d-q reference frame can be described as follows:

$$
\begin{gathered}
V_{s d}=R_{s} I_{s d}+\frac{d \psi_{s d}}{d t}-\omega_{r} \psi_{s q} \\
V_{s q}=R_{s} I_{s q}+\frac{d \psi_{s q}}{d t}+\omega_{r} \psi_{s d} \\
\psi_{s d}=L_{s} I_{s d}+\psi_{f} \\
\psi_{s q}=L_{s} I_{s q} \\
T_{e}=\frac{3}{2} p \psi_{f} I_{s q}
\end{gathered}
$$

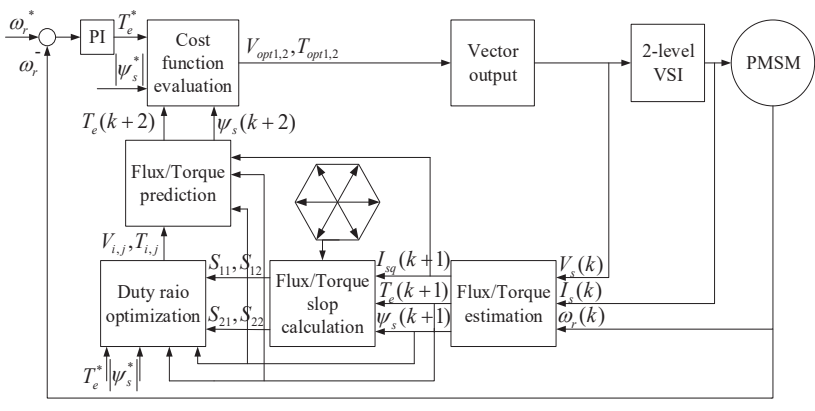

Fig. 3. Diagram of the proposed MPTC strategy.

where $V_{s d}$ and $V_{s q}$ are $\mathrm{d}$-axis and q-axis stator voltage respectively, $\psi_{s d}$ and $\psi_{s q}$ are d-axis and q-axis stator flux respectively, $I_{s d}$ and $I_{s q}$ are d-axis and q-axis stator current respectively, $T_{e}$ is electromagnetic torque, $p$ is the number of pole pairs, $R_{s}$ is stator resistance, $L_{s}$ is inductance, $\omega_{r}$ is electrical rotor speed, $\psi_{f}$ is permanent magnet flux linkage.

In this paper, a traditional two-level voltage source inverter (VSI) is used to drive the PMSM, as shown in Fig. 1, and the generated voltage vectors, shown in Fig. 2, can be expressed as follow:

$$
V_{j}=\frac{2}{3} V_{d c}\left(S_{a}+e^{i 2 \pi / 3} S_{b}+e^{i 4 \pi / 3} S_{c}\right)
$$

where $V_{j}(j=0, \ldots, 7)$ represent the available voltage vectors, $S_{a}, S_{b}, S_{c}$ denote the switching states of the three inverter arms.

\section{Proposed MPTC StRATEgY}

The diagram of the proposed MPTC strategy is shown in Fig. 3 , it consists of flux and torque estimation, calculation of flux and torque slope, duty ratio optimization, flux and torque prediction, cost function evaluation and vector output.

\section{A. State Estimation}

In practical implementation, due to the one period delay between the selected voltage vector and the applied voltage vector, the estimation of flux and torque for the next period is required. Based on the discrete model of PMSM and the variables of the motor, the stator flux and the torque at the next sampling instant can be estimated. For this purpose, vector combination and corresponding durations determined in the previous control period are utilized. The prediction of flux and torque can be expressed as:

$$
\begin{gathered}
\psi_{s}(k+1)=\psi_{s}(k)+T_{1}(k) V_{s 1}(k)+T_{2}(k) V_{s 2}(k)-T_{s} R_{s} I_{s}(k) \\
T_{e}(k+1)=\frac{3}{2} p \psi_{f} I_{s q}(k+1)
\end{gathered}
$$




$$
\begin{gathered}
I_{s q}(k+1)=I_{s q}(k)+\frac{T_{s}}{L_{s}}\left[-L_{s} \omega_{r}(k) I_{s d}(k)-R_{s} I_{s q}(k)-\omega_{r}(k) \psi_{f}\right] \\
+\frac{1}{L_{s}}\left[T_{1}(k) V_{s q 1}(k)+T_{2}(k) V_{s q 2}(k)\right]
\end{gathered}
$$

where $\psi_{s}=\left[\psi_{s d}, \psi_{s q}\right]^{T}, I_{s}=\left[I_{s d}, I_{s q}\right]^{T}, V_{s}=\left[V_{s d}, V_{s q}\right]^{T}, T_{s}$ is a whole control period, $V_{s 1}$ and $V_{s 2}$ are the first and the second optimal voltage vector, which are determined in the last control period, $T_{1}$ and $T_{2}$ are their time durations respectively. $T_{1} \leq T_{s}$ and $T_{2}=T_{s}-T_{1}$.

\section{B. Construction of Possible Inputs}

- Calculation of flux slope and torque slope

For MPTC, torque and stator flux need to be predicted under every voltage vector. Thus, torque variations and flux variations due to different vectors are required to be deduced, which are important for duty ratio optimization. Based on (1)-(4), the flux derivative under voltage vector can be expressed as:

$$
\frac{d\left|\psi_{s}\right|}{d t}=\frac{\psi_{s} \cdot\left(V_{s}-R I_{s}\right)}{\left|\psi_{s}\right|}
$$

As mentioned before, the whole control period is equal to $T_{s}$ and the time durations for the first and second voltage vector are $T_{1}$ and $T_{2}$, flux variation under the two voltage vectors can be represented as:

$$
\begin{gathered}
\Delta\left|\psi_{s 1}\right|=\frac{\psi_{s} \cdot\left(V_{s 1}-R I_{s}\right)}{\left|\psi_{s}\right|} T_{1} \triangleq S_{11} T_{1} \\
\Delta\left|\psi_{s 2}\right|=\frac{\psi_{s} \cdot\left(V_{s 2}-R I_{s}\right)}{\left|\psi_{s}\right|} T_{2} \triangleq S_{12} T_{2}
\end{gathered}
$$

respectively, where $S_{11}=\frac{\psi_{s} \cdot\left(V_{s 1}-R I_{s}\right)}{\left|\psi_{s}\right|}, S_{12}=\frac{\psi_{s} \cdot\left(V_{s 2}-R I_{s}\right)}{\left|\psi_{s}\right|}$, denote the flux slopes under the first and the second voltage vector respectively.

Similarly, according to (1)-(5), the torque derivative and the torque variation under the two voltage vectors can be expressed as:

$$
\begin{gathered}
\frac{d T_{e}}{d t}=\frac{3 p \psi_{f}}{2 L_{s}}\left(V_{s q}-R_{s} I_{s q}-\omega_{r} \psi_{s d}\right) \\
\Delta T_{e 1}=\frac{3 p \psi_{f}}{2 L_{s}}\left(V_{s q 1}-R_{s} I_{s q}-\omega_{r} \psi_{s d}\right) T_{1} \triangleq S_{21} T_{1} \\
\Delta T_{e 2}=\frac{3 p \psi_{f}}{2 L_{s}}\left(V_{s q 2}-R_{s} I_{s q}-\omega_{r} \psi_{s d}\right) T_{2} \triangleq S_{22} T_{2}
\end{gathered}
$$

respectively, where

$$
\begin{aligned}
& S_{21}=\frac{3 p \psi_{f}}{2 L_{s}}\left(V_{s q 1}-R_{s} I_{s q}-\omega_{r} \psi_{s d}\right) \\
& S_{22}=\frac{3 p \psi_{f}}{2 L_{s}}\left(V_{s q 2}-R_{s} I_{s q}-\omega_{r} \psi_{s d}\right)
\end{aligned}
$$

are the torque slopes under the first and the second voltage vector respectively. It should be noticed that the control period $T_{s}$ is small enough to assume all the variables are constant within a control period. Therefore, both flux slope and torque slope can be regarded as fixed value within one control period.

- Duty ratio optimization

For two-vector MPTC, determining the switching instant is the key issue. In [11] and [13], deadbeat torque control principle is used to nullify the torque tracking error at the end of the next control period, which cannot lead to good performance of mean torque. In addition, it failed to take flux error into account. In this paper, a RMS-based principle is employed. It aims at reducing the RMS error of both torque and stator flux during the entire control period, and the RMS error can be expressed as [4]:

$$
\begin{aligned}
E= & \frac{\lambda_{\psi}}{T_{s}} \int_{0}^{T_{1}}\left(\psi_{s}^{e}+s_{11} t\right)^{2} d t+\frac{\lambda_{\psi}}{T_{s}} \int_{T_{1}}^{T_{s}}\left[\psi_{s}^{e}+s_{11} t+s_{12}\left(t-T_{1}\right)\right]^{2} d t \\
& +\frac{1}{T_{s}} \int_{0}^{T_{1}}\left(T_{e}^{e}+s_{21} t\right)^{2} d t+\frac{1}{T_{s}} \int_{T_{1}}^{T_{s}}\left[T_{e}^{e}+s_{21} t+s_{22}\left(t-T_{1}\right)\right]^{2} d t
\end{aligned}
$$

where $\lambda_{\psi}$ is a weighting factor of stator flux, $\psi_{s}^{e}=\psi_{s}-\psi_{s}^{*}$, $T_{e}^{e}=T_{e}-T_{e}^{*}$ are the initial flux and torque errors at the beginning of the control period respectively. Therefore, letting $d E / d T_{1}=0$, the time duration for the first voltage vector is deduced as:

$$
T_{1}=\frac{-B+\sqrt{B^{2}-4 A C}}{2 A}
$$

where

$$
\begin{gathered}
A=-\lambda_{\psi} s_{1}\left(s_{11}+s_{1}\right)-s_{2}\left(s_{21}+s_{2}\right) \\
B=2 \lambda_{\psi} s_{1}\left(T_{s} s_{1}-\psi_{s}^{e}\right)+2 s_{2}\left(T_{s} s_{2}-T_{e}^{e}\right) \\
C=T_{s} \lambda_{\psi} s_{1}\left(T_{s} s_{12}+2 \psi_{s}^{e}\right)+T_{s} s_{2}\left(T_{s} s_{22}+2 T_{e}^{e}\right)
\end{gathered}
$$

$S_{1}=S_{11}-S_{12}, S_{2}=S_{21}-S_{22}$. In this paper, as two vectors applied in one control period, the corresponding time duration should be calculated for every vector combination before prediction. Different from the method [11], [12], which determines the time duration after cost function evaluation, this method guarantees both the vector and the time duration can be evaluated in the cost function.

\section{Prediction and cost function minimization}

For two-vector MPTC, some prior strategies use zero vector as the second, which achieve limited torque performance 
improvement, since zero vector may not be the optimal one for the second vector. Conversely, some strategies select the second vector from all the seven voltage vectors, which leads to high switching frequency and increasing computational burden. To reach a balance, this paper uses an active vector as the first vector. For the second vector, the two adjacent vectors of the first one and zero vector are considered as the candidates. Thus, $6 \times 3=18$ vectors combinations are provided.

Based on the estimations and the calculated time duration for the $(\mathrm{k}+1)$ th control period, flux and torque at the $(\mathrm{k}+2)$ th sampling instant under all vector combinations can be predicted as:

$$
\begin{gathered}
\psi_{s \mid n}(k+2)=\psi_{s}(k+1)+T_{1}(k+1) V_{s \mid i}(k+1) \\
+T_{2}(k+1) V_{s 2 \mid j}(k+1)-T_{s} R_{s} I_{s}(k+1) \\
T_{e \mid n}(k+2)=\frac{3}{2} p \psi_{f} I_{s q \mid n}(k+2) \\
I_{s q \mid n}(k+2)=\frac{T_{s}}{L_{s}}\left[-L_{s} \omega_{r}(k+1) I_{s d}(k+1)-R_{s} I_{s q}(k+1)-\omega_{r}(k+1) \psi_{f}\right] \\
+\frac{1}{L_{s}}\left[T_{1}(k+1) V_{s q \mid i}(k+1)+T_{2}(k+1) V_{s q \mid j}(k+1)\right]+I_{s q}(k+1)
\end{gathered}
$$

where $n=1,2, \ldots, 18$, represents the number of the vector combination, $i=1,2, \ldots, 6$ is the number of the first vector, $j=i-1, i+1,0$, is the number of the second vector. The vector combination, which can minimize a cost function will be selected as the optimal vector combination and applied in next control period. In this paper, to be coincident with the duty ratio optimization principle, the cost function is expressed as:

$$
g=\left[T_{e}^{*}-T_{e}(k+2)\right]^{2}+k_{\psi}\left[\left|\psi_{s}^{*}\right|-\left|\psi_{s}(k+2)\right|\right]^{2}
$$

where $k_{\psi}$ is the weight coefficient of stator flux.

\section{SimUlATION RESULTS}

The proposed MPTC is verified by simulation in the environment of Matlab/Simulink, the parameters of the motor are listed in Table 1. The performance of conventional FCSMPTC [7], MPTC with torque deadbeat solution (DB-MPTC) [11] and the proposed MPTC will be compared. For the FCSMPTC, one active voltage vector is applied in a fixed control period and the sampling frequency is $10 \mathrm{kHz}$. The sampling frequency of the DB-MPTC and the proposed MPTC is set to $5 \mathrm{kHz}$. It should be noticed that the sampling frequency of the FCS-MPTC strategy is twice as high as other two MPTC strategies. Since one vector is applied in one control period for the FCS-MPTC, while two vectors are applied for the other two MPTC strategies, it is reasonable to set this inequality. The waveforms of torque, flux, and phase current for the three strategies are shown in Fig. 4.

In Fig. 4, the load torque is suddenly changed from $10 \mathrm{Nm}$ to $11 \mathrm{Nm}$ at $\mathrm{t}=0.15 \mathrm{~s}$ to show the dynamic performance of the proposed MPTC strategy. It can be easily seen in Fig. 4(a) and Fig.4 (b) that lower torque ripple and flux ripple are achieved in
TABLE I. MOTOR PARAMETERS

\begin{tabular}{|c|c|c|}
\hline Motor parameter & & Value \\
\hline DC voltage & $V_{d c}$ & $200 \mathrm{~V}$ \\
\hline Number of pole pairs & $p$ & 1 \\
\hline Permanent magnet flux linkage & $\psi_{f}$ & $1 \mathrm{~Wb}$ \\
\hline Stator resistance & $R_{s}$ & $1.91 \Omega$ \\
\hline Inductance & $L_{s}$ & $0.016 \mathrm{H}$ \\
\hline Flux amplitude reference & $\left|\psi_{s}^{*}\right|$ & $1.0227 \mathrm{~Wb}$ \\
\hline
\end{tabular}
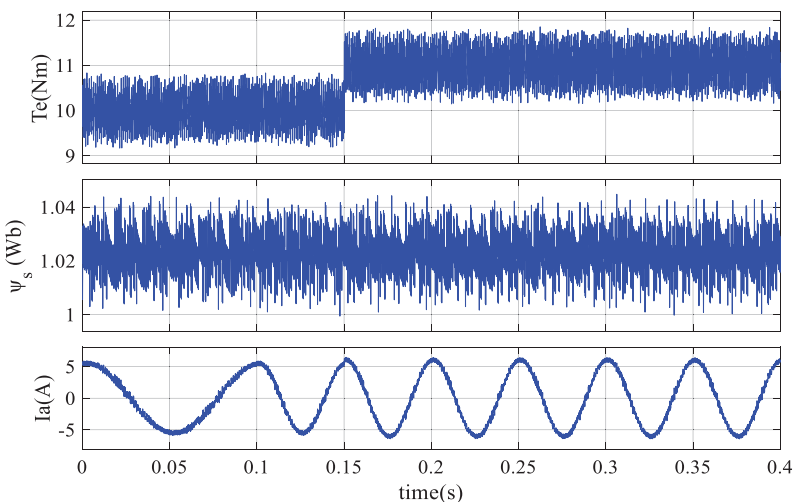

(a)
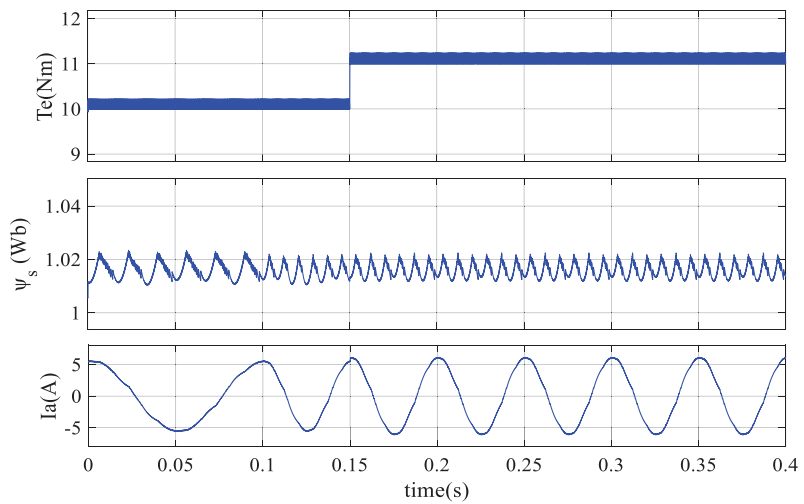

(b)

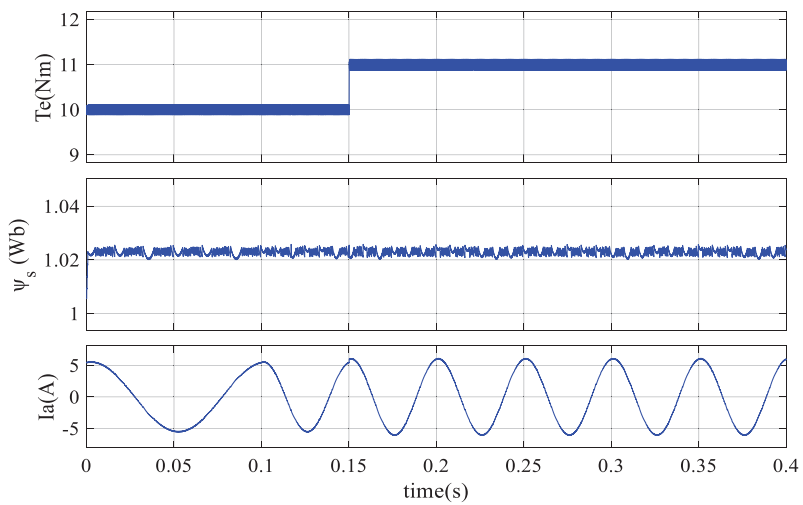

(c)

Fig. 4. Waveforms of torque, stator flux and phase current. (a) waveforms of FCS-MPTC (b) waveforms of DB-MPTC (c) waveforms of the proposed MPTC 

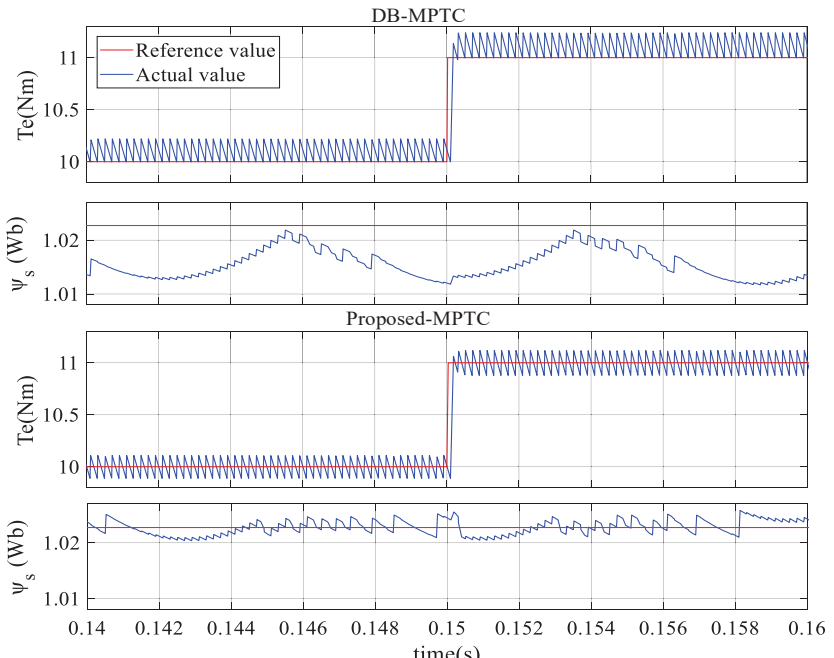

Fig.5. Tracking trajectory comparation.

TABLE II. COMPARATION OF STEADY STATE PERFORMANCE

\begin{tabular}{|c|c|c|c|}
\hline Parameter & FS-MPTC & DB-MPTC & $\begin{array}{c}\text { Proposed } \\
\text { MPTC }\end{array}$ \\
\hline$T_{r i p}$ & $0.3024 \mathrm{Nm}$ & $0.1386 \mathrm{Nm}$ & $0.0701 \mathrm{Nm}$ \\
\hline$\psi_{r i p}$ & $0.00667 \mathrm{~Wb}$ & $0.00736 \mathrm{~Wb}$ & $0.00113 \mathrm{~Wb}$ \\
\hline
\end{tabular}
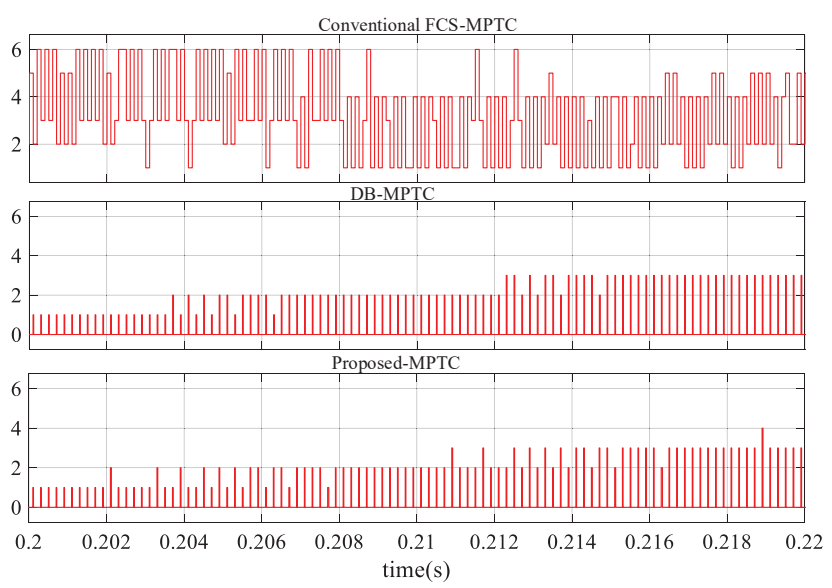

Fig.6. Switching states of the three strategies.

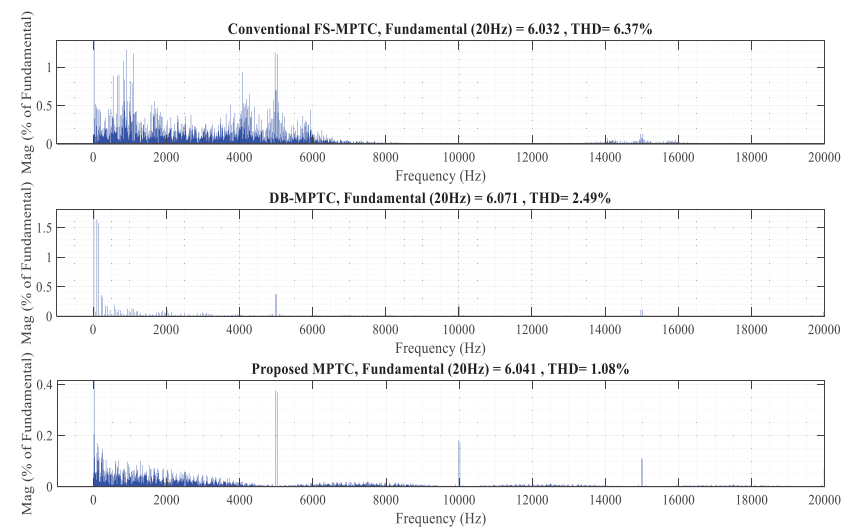

Fig.7. Harmonic spectrum of phase current for the three strategies.
DB-MPTC than that in FCS-MPTC. However, since torque deadbeat solution is utilized, the torque of DB-MPTC can only reach the reference value at the end of every control period. For the proposed MPTC, an improved flux and torque RMS solution is employed. Hence, the torque error can be minimized during the whole control period, which can be easily seen in Fig.4 (c). Furthermore, it can be seen in Fig. 4(b) that DB-MPTC has bad performance in flux tracking, because it selects the active vector before duty ratio optimization. Meanwhile, the deadbeat solution does not consider the flux ripple when determining the duty ratio. Conversely, the proposed MPTC calculates the duty ratio for all possible vector combinations before cost function evaluation with taking the flux error into account. Therefore, the proposed MPTC achieves good performance of flux tracking, as shown in Fig. 4(c).

Fig. 5 shows the tracking trajectories of the DB-MPTC and the proposed MPTC. It can be clearly seen that the proposed MPTC strategy performs better than the DB-MPTC in terms of torque and flux steady state performance. The mean torque error is significantly reduced and the tracking performance of stator flux is much improved. The comparative results of torque and flux ripple under the three MPTC strategies are listed in Table 2, which are calculated based on the following equations:

$$
\left\{\begin{array}{l}
T_{\text {rip }}=\sqrt{\frac{1}{n} \sum_{i=1}^{n}\left(T_{e}(i)-T_{e}^{*}\right)^{2}} \\
\psi_{\text {rip }}=\sqrt{\frac{1}{n} \sum_{i=1}^{n}\left(\psi_{s}(i)-\psi_{s}^{*}\right)^{2}}
\end{array}\right.
$$

where $n$ represents the sampling number.

Fig. 6 shows the switching states of the three strategies. It can be seen that different switching states are generated in the proposed MPTC strategy to produce better steady state performance. In addition, the harmonic spectrum of phase current under the three strategies is shown in Fig .7. It can be seen that better current harmonic spectrum can be obtained in the proposed MPTC, which further proves the effectiveness of the proposed MPTC strategy.

\section{CONCLUSION}

This paper proposes an improved two-vector MPTC with RMS duty ratio optimization. This method utilizes the principle of RMS error in determining the duty ratio. Thus, Lower average torque ripple and better current harmonic spectrum are achieved. Meanwhile, since the flux error is also considered in the RMS solution, better flux tracking performance as well as lower flux ripple are obtained. Besides, this strategy evaluates the duty ratio of every vector combination in cost function, which can improve the steady state performance. In addition, due to the restriction on the second vector, fixed low switching frequency with low computational burden are achieved.

\section{REFERENCES}

[1] M. H. Vafaie, B. M. Dehkordi, P. Moallem and A. Kiyoumarsi, "Improving the Steady-State and Transient-State Performances of PMSM Through an Advanced Deadbeat Direct Torque and Flux Control System," IEEE Trans. Power Electron., vol. 32, no. 4, pp. 2964-2975, Apr. 2017. 
[2] Y. Zhang, D. Xu, and L. Huang, "Generalized Multiple-Vector-Based Model Predictive Control for PMSM Drives,” IEEE Trans. Ind. Electron., vol. 65, no. 12, pp. 9356-9366, 2018.

[3] P. Kakosimos and H. Abu-Rub, "Predictive Speed Control With Short Prediction Horizon for Permanent Magnet Synchronous Motor Drives," IEEE Trans. Power Electron., vol. 33, no. 3, pp. 2740-2750, 2018.

[4] F. Niu, K. Li, and Y. Wang, "Direct Torque Control for PermanentMagnet Synchronous Machines Based on Duty Ratio Modulation," IEEE Trans. Ind. Electron., vol. 62, no. 10, pp. 6160-6170, Oct. 2015.

[5] Y. Wang, X. Wang, W. Xie, F. Wang, M. Dou, R. M. Kennel, et al., "Deadbeat Model-Predictive Torque Control With Discrete Space-Vector Modulation for PMSM Drives," IEEE Trans. Ind. Electron., vol. 64, no. 5, pp. 3537-3547, May 2017.

[6] Y. Cho, Y. Bak, and K.-B. Lee, "Torque-Ripple Reduction and Fast Torque Response Strategy for Predictive Torque Control of Induction Motors," IEEE Trans. Power Electron., vol. 33, no. 3, pp. 2458-2470, 2018.

[7] H. Miranda, P. Cortes, J. Yuz, and J. Rodriguez, "Predictive torque control of induction machines based on state-space models," IEEE Trans. Ind. Electron., vol. 56, no. 6, pp. 1916-1924, Jun. 2009.
[8] M. Siami, D. Arab Khaburi, and J. Rodriguez, "Simplified Finite Control Set-Model Predictive Control for Matrix Converter-Fed PMSM Drives," IEEE Trans. Power Electron., vol. 33, no. 3, pp. 2438-2446, 2018.

[9] M. Amiri, J. Milimonfared, and D. A. Khaburi, "Predictive Torque Control Implementation for Induction Motors Based on Discrete Space Vector Modulation," IEEE Trans. Ind. Electron., vol. 65, no. 9, pp. 68816889, 2018.

[10] M. Pacas and J. Weber, "Predictive direct torque control for the PM synchronous machine," IEEE Trans. Ind. Electron., vol. 52, no. 5,pp. 1350-1356, Oct. 2005.

[11] Y. Xu, Q. Zhou and B. Zhang, "A Model Predictive Torque Control Strategy of PMSM with Torque Deadbeat Duty Cycle Control,” IEEE Int. Power. Elec., 2016, pp. 782-785.

[12] Y. Zhang and H.Yang, "Torque ripple reduction of model predictive torque control of induction motor drives," in Proc. IEEE Energy Convers. Congr.Expo., 2013, pp. 1176-1183.

[13] Y. Zhang, and H. Yang, "Generalized Two-Vector-Based ModelPredictive Torque Control of Induction Motor Drives," IEEE Trans. Power Electron., vol. 30, no. 7, pp. 3818-3829, Jul. 2015. 